\title{
Averaging Temporal Duration and Spatial Position
}

\author{
Ken Cheng \\ University of Toronto
}

\author{
Marcia L. Spetch \\ University of Alberta
}

\author{
Paul Miceli \\ University of Toronto
}

\begin{abstract}
Pigeons and humans performed on a task in which spatial position and elapsed time redundantly signaled the availability of reward. On each training trial, a landmark moved steadily across a monitor screen. After a fixed amount of time and movement, reward was available for a response. On occasional unrewarded tests, the landmark moved at $0.50,0.75$, $1.00,1.50$, or 2.00 times the training speed. In both pigeons and humans, the central tendency in the response distribution on tests differed across speeds, when measured in terms of both elapsed time and landmark position. Pigeons and humans seem to average a duration of time and a spatial position to find a single criterion time-place corresponding to the expected time-place of reward.
\end{abstract}

In deciding when to engage in a task, an animal usually has a number of cues indicating the appropriate time for action. In doing a task at a particular time during the day, for example, the animal typically can rely on its internal circadian clock as well as external cues such as the position of the sun (see Gallistel, 1990, for a review). On a time scale shorter than the time during a day, the timing of events is also usually indicated by multiple cues. Consider, for example, a pet cat waiting for the arrival of food after hearing the electric can opener operating. The food will arrive approximately at a fixed time after the onset of the sound, so that its internal interval clock can be used to estimate food arrival. But other cues in the actions of the person feeding the cat also indicate the time of arrival of food. In deciding where to search for a desired object, multiple cues also indicate the location to search. For instance, different landmarks might be used to pinpoint the target location.

Laboratory studies indicate that when multiple cues are available in the temporal and spatial domains, an animal will often average the dictates of different cues (Cheng, 1992). For example, in one spatial search task pigeons were looking for hidden food within a square arena covered with wood chips (Cheng, 1988). The goal was at a particular place within the arena, located in front of a piece of blue cardboard. When the cardboard was shifted on tests, the

Ken Cheng and Paul Miceli, Department of Psychology, University of Toronto, Toronto, Ontario, Canada; Marcia L. Spetch, Department of Psychology, University of Alberta, Edmonton, Alberta, Canada.

This work was supported by grants from the Natural Sciences and Engineering Research Council of Canada.

Correspondence concerning this article should be addressed to Ken Cheng, who is now at the School of Behavioural Sciences, Macquarie University, Sydney NSW 2109 Australia, or to Marcia L. Spetch, Department of Psychology, University of Alberta, Edmonton, Alberta, Canada T6G 2E9. Electronic mail may be sent via Internet to kcheng@bunyip.bhs.mq.edu.au or to mspetch@ psych.ualberta.ca. birds searched most at a location in between the goal location as dictated by the cardboard and the goal location as dictated by the rest of the (unshifted) landmarks. Numerous other experiments with pigeons in search tasks, both on the laboratory floor (Cheng, 1989, 1994; Cheng \& Sherry, 1992 ) and on the surface of a monitor (Spetch, Cheng, \& Mondloch, 1992), corroborate this point.

In interval timing, Cheng and Roberts (1991) tested pigeons on the peak procedure with multiple cues. In the peak procedure, two types of trials are given. On training trials, which constitute most of the trials, a signal indicates the start of a trial. After a fixed interval (FI) since signal onset, a response is rewarded. On occasional test trials, the signal comes on as usual, but it stays on for a long period and then is extinguished. No reward is given, no matter how the subject responds. On tests, the subject typically makes a burst of responding at a high rate, called a run, around the time of the FI when food is expected (Cheng \& Westwood, 1993; Cheng, Westwood, \& Crystal, 1993; Church, Meck, \& Gibbon, 1994; Gibbon \& Church, 1990). When the distribution of responses in time since signal onset are pooled over many trials, a Gaussian shape is obtained with the peak (peak time) near the FI. Cheng and Roberts (1991) trained pigeons on a peak procedure with two separate signals associated with different FIs. On tests with both signals presented together, the birds peaked at a time in between the two FIs, indicating that they had averaged the dictates of the two signals. Cheng and Roberts (1989) found a similar instance of averaging in interval timing. Recently, Fetterman (1993) also found pigeons using both timing and counting in a single task, thus averaging temporal and numerical dictates. Counting, however, has been suggested to be a part of the timing system (Meck \& Church, 1983).

In this study we used a variant of the peak procedure, which we call the spatiotemporal peak procedure, to determine whether pigeon and human participants averaged the dictates of two very different domains, namely time and space. The signal in the task consisted of a graphic object 
(white rectangle) that moved at a constant speed on a monitor. As a result, the availability of reward was given not only by an FI of time but also by the spatial position of the moving object. Specifically, on a trial the rectangle starts moving across the screen at $1 \mathrm{~cm} / \mathrm{s}$. After $10 \mathrm{~s}$ and $10 \mathrm{~cm}$ for some participants and $8 \mathrm{~s}$ and $8 \mathrm{~cm}$ for others, a response is rewarded. Participants can use either the passage of time or the position of the rectangle to recognize when reward is available. On occasional tests, the rectangle moves across the screen at a constant speed that is sometimes different from that used in training. Consider, for example, the case in which it moves at twice the speed (2 cm/s). A participant following solely the dictates of interval timing would show peak responding at about the same time as on control tests (i.e., for participants on FI $10 \mathrm{~s}$, peak responding would occur around $10 \mathrm{~s}$ since signal onset). But a participant following solely the dictates of the spatial position of the rectangle would show peak responding much earlier, at approximately the time when the rectangle reaches its usual position where reward becomes available, in this case around $5 \mathrm{~s}$. A participant averaging the dictates of space and time, however, would show peak responding at a point in between these two durations. And should a participant show such an intermediate peak time, this would suggest that the participant had somehow averaged the dictates of two separate systems, an interval-timing system and some system processing information on spatial positions.

Such a compromise reveals something interesting about the brain. It indicates that different systems or modules within the brain dealing with fundamentally different aspects of the world, space and time, can be working in tandem on a task. It indicates that dictates couched in terms of spatial positions and dictates couched in terms of temporal durations must at some point be translated into a common code that can be averaged.

We used pigeons on this task because they have been traditional subjects in experiments on timing and a large body of data exists about them. Human interval timing shares much with that of rats and pigeons (e.g., Wearden, 1991, 1995; Wearden \& McShane, 1988). But the peak procedure has been used little with humans (Hinton, Gibbon, Rakitin, \& Meck, 1993). In comparing humans with pigeons, we are not only testing the generality of the finding of interest but also attempting a version of the peak procedure with humans. With the touch-screen apparatus, human participants can be tested on the same task with but few modifications, making data gathering efficient.

\section{Method}

\section{Participants}

The pigeons were 4 White Kings, each experienced at touchscreen tasks and at timing tasks. They were housed individually in large cages with free access to water and grit on a 14:10 light--dark cycle (lights on at 6:00 a.m.). They were maintained at approximately $85 \%$ of their free feeding weight by pellets received during experimental sessions and mixed grain received after experimental sessions.

The humans were undergraduate students who participated in the experiment for credit in their introductory psychology course. One man and 5 women, ranging from 18 to 23 years of age, were tested in Group FI 10. Three men and 7 women, ranging from 18 to 51 years of age, were tested in Group FI 8.

\section{Apparatuses}

The birds were tested in two custom-built chambers $(35 \mathrm{~cm}$ long $\times 30 \mathrm{~cm}$ wide $\times 45 \mathrm{~cm}$ high). The floor of the chambers (parallel bars) were raised $4 \mathrm{~cm}$ from the table on which the chamber stood. At one end was an opening large enough for the entire surface of the Zenith 1492 monitor with attached Carroll Touch 1492 Smart Frame (Carroll Touch, Little Rock, TX). The size of the monitor was $27 \mathrm{~cm}$ wide $\times 19 \mathrm{~cm}$ high. At the floor to the front, a stand, that was $5 \mathrm{~cm}$ wide $\times 6 \mathrm{~cm}$ high from the floor of the chamber, was constructed. On this stand was a hopper into which a Gerbrands feeder delivered pellets from the left side. The reward delivered was three $20-\mathrm{mg}$ pigeon pellets (P. J. Noyes, Inc., Arlington, VA). A 7-watt light on the ceiling above the hopper lit up during food delivery until $4 \mathrm{~s}$ after the last pellet was delivered. Microcomputers, located in an adjacent room, controlled experimental contingencies and recorded peck coordinates and times.

The humans were tested in a small, private room that contained a computer equipped with a touch-screen. The computer monitor (Zenith 1490) and touch-screen (Carroll Touch 1490 Smart Frame) provided the same search space and stimuli as used for pigeons. Participants sat in a chair in front of the monitor and searched by touching the screen with the eraser end of a pencil.

\section{Procedure for Pigeons}

The birds were first autoshaped to peck at a warning stimulus for reward. The warning stimulus was a $2.0-\mathrm{cm}$ red square with its center located $6.5 \mathrm{~cm}$ from the bottom and $12.5 \mathrm{~cm}$ from the left.

During the experiment, all sessions were 100 trials long or a maximum of $1.5 \mathrm{hr}$. Intertrial intervals were $10 \mathrm{~s}$ throughout. The birds were first given one pretraining session. On each trial, the warning stimulus appeared. When it was pecked once, it disappeared and was replaced with the target stimulus. This was a green isosceles triangle, $2.0 \mathrm{~cm}$ across at the base and $2.0 \mathrm{~cm}$ high, with its center located $7.0 \mathrm{~cm}$ from the bottom and $12.5 \mathrm{~cm}$ from the left. Pecking at the target terminated the display and resulted in the delivery of reward. The effective area that was programmed for the warning and target stimulus was actually a $8.0 \mathrm{~cm} \times 8.0 \mathrm{~cm}$ square, so that any peck near the stimulus would be registered.

The birds were next given 12 training sessions. On each trial, the warning stimulus first appeared. A peck at it replaced it with the stimulus display (Figure 1). This consisted of the target stimulus and a moving white rectangle at the top left of the screen. The rectangle measured $1.2 \mathrm{~cm}$ wide and $2.0 \mathrm{~cm}$ high, and its center was $5.0 \mathrm{~cm}$ from the top. The rectangle moved to the right at a rate of $1 \mathrm{~cm} / \mathrm{s}$ until a reward was earned or until it disappeared off the right edge. A reward was programmed for the first peck at the target (the stationary green triangle) after $10 \mathrm{~s}$. Pecking at the moving rectangle had no consequences. During casual observations, no bird pecked regularly at this stimulus.

The birds were next given five test sessions with control tests, in order to accustom them to unrewarded trials. Every block of four trials contained a test, but tests were not given on consecutive trials. On a control test, the warning stimulus first appeared as 
Moving Landmark

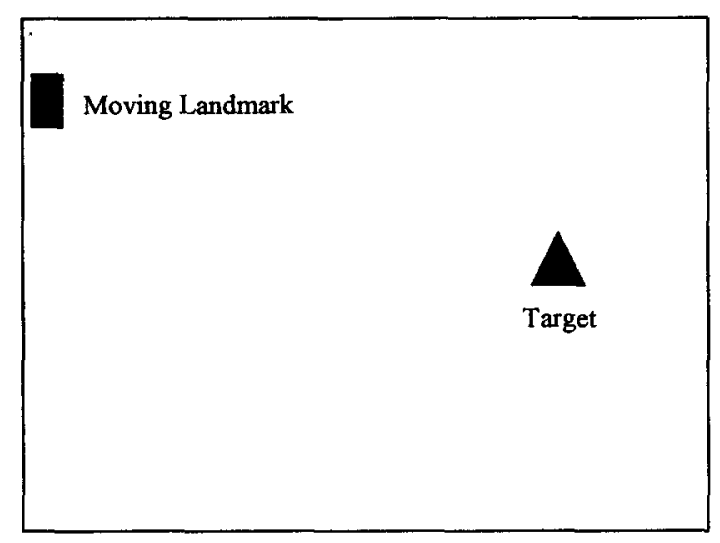

Figure 1. The stimulus display as it appeared at the start of a trial, after the participant has touched the warning stimulus. The landmark at the top left moved from left to right while responses on the green triangle were registered. The exact locations of the target and landmark varied for the pigeons and two groups of humans.

usual. A peck at it replaced it with the stimulus display, as on training trials. The white rectangle moved to the right at the usual speed for $40 \mathrm{~s}$, after which the test ended, with no reward delivered. The times of the pecks made by the bird were recorded.

The birds were next given 30 test sessions with different tests. These sessions resembled the previous phase except that five different kinds of tests were given, five each in each session. The tests only differed in the speed at which the white rectangle moved across the screen. The speeds $(\mathrm{cm} / \mathrm{s})$ on tests were $0.50,0.75,1.00$, 1.50 , and 2.00 .

To ensure that behavior was asymptotic, Sessions 11-30 were used for data. The number of pecks in each 1-s bin after the appearance of the white rectangle was totaled for each bird in each test condition. From these distributions, a time of peak pecking (peak time) was calculated by the median iteration procedure used in many such studies (e.g., Cheng \& Westwood, 1993; S. Roberts, 1981; W. A. Roberts, Cheng, \& Cohen, 1989).

\section{Procedure for Humans}

At the beginning of each session, participants were provided with the following information: Their task would be to try to earn points by touching the green triangle. A prize of 100 points would be available on some randomly selected trials. A cost of 1 point would be incurred for every touch. Participants were told to "try to figure out the conditions under which you can obtain the prize." No mention was made of spatial or temporal factors.

The experimenter asked the participant to write down his or her age and sex on a piece of paper and then ran a demonstration program that presented one training trial. The experimenter showed the participant how to touch the screen with a pencil. Any procedural questions posed by the participant were answered with the statement, "I'm sorry, but I cannot reveal any other information about the procedure as it may influence the way you perform." After obtaining confirmation that the participant still wished to participate, the experimenter then started the program for the participant and left the room.

The procedure on training and test trials paralleled those for pigeons. The participant's program began with three training trials. On each training trial, the warning stimulus, with a center $9 \mathrm{~cm}$ from the bottom and $13 \mathrm{~cm}$ from the left, first came on. A touch at it produced the experimental display. In the display for the first 6 participants tested (Group FI 10), the center of the green triangle (target) was $9.5 \mathrm{~cm}$ from the bottom and $13.0 \mathrm{~cm}$ from the left, and the center of the white rectangle (moving landmark) was $3.0 \mathrm{~cm}$ from the top. The display for the last 10 participants tested (Group FI 8) was identical except that the target was 20.0 rather than 13.0 $\mathrm{cm}$ from the left. For participants in Group FI 10, reinforcement in the form of a 100-point prize was available for the first touch at the target $10 \mathrm{~s}$ after the beginning of the display, which was accompanied by a $10.0-\mathrm{cm}$ advancement of the moving landmark toward the right. For participants in Group FI 8, the 100-point prize was available for the first touch at the target $8 \mathrm{~s}$ after the beginning of the display, which was accompanied by an $8.0-\mathrm{cm}$ advancement of the moving landmark. In all other ways, the procedure was identical for the two groups. The reinforcement display indicated the 100 -point prize, as well as the total points earned to date, the total points used to date, and the net total points earned to date. The display stayed on for $3 \mathrm{~s}$, and then the warning stimulus for the next trial appeared. After the first 3 training trials, participants received two blocks of 3 trials in which each block contained one control test and 2 training trials. These two tests were not used for data. Then participants completed 3 blocks of 15 trials. In each block was one test of each type and 10 training trials. The same test types (five speeds) that were given to pigeons were given to humans (three of each type). All tests lasted for $40 \mathrm{~s}$ and terminated with a display that read, "No points earned on this trial," with information about the total points earned to date, total points used to date, and net points earned to date. The median time of response on each test type for each participant was used for data (i.e., peak time was the median response time). In reporting the results, the alpha level of significance was set at 0.05 throughout.

\section{Results}

The average peak times for the birds and humans are shown in Figure 2, top panel. The same data are translated to peak places (i.e., the position of the moving landmark at peak time) and shown in the bottom panel of Figure 2. The data from 4 human participants in Group FI 8 condition were excluded because the peak times on the control tests exceeded twice the value of the FI. In all groups of participants for both time and space, the data appear linear. A best-fitting regression line (least-squares criterion) has been drawn through each set of data points.

Statistical analyses, summarized in Table 1, confirm these impressions. In each data set for both time and space, a within-subjects analysis of variance on peak times and peak places reveals significant differences across conditions. Furthermore, a trend analysis with unequal intervals (Grandage, 1958) shows highly significant linear trends but no significant higher order trends. A linear regression line accounts for most of the variance, and errors around the regression line appear unsystematic, as testified by the lack of significant higher order trends. Thus, the data fall into the pattern of a straight line with a nonzero slope. In every case, peak times vary linearly with $1 /$ speed, and peak places vary linearly with speed. Exclusive use of position in determining criterion would produce constant peak places across conditions, whereas exclusive use of time in determining criterion would produce constant peak times across condi- 

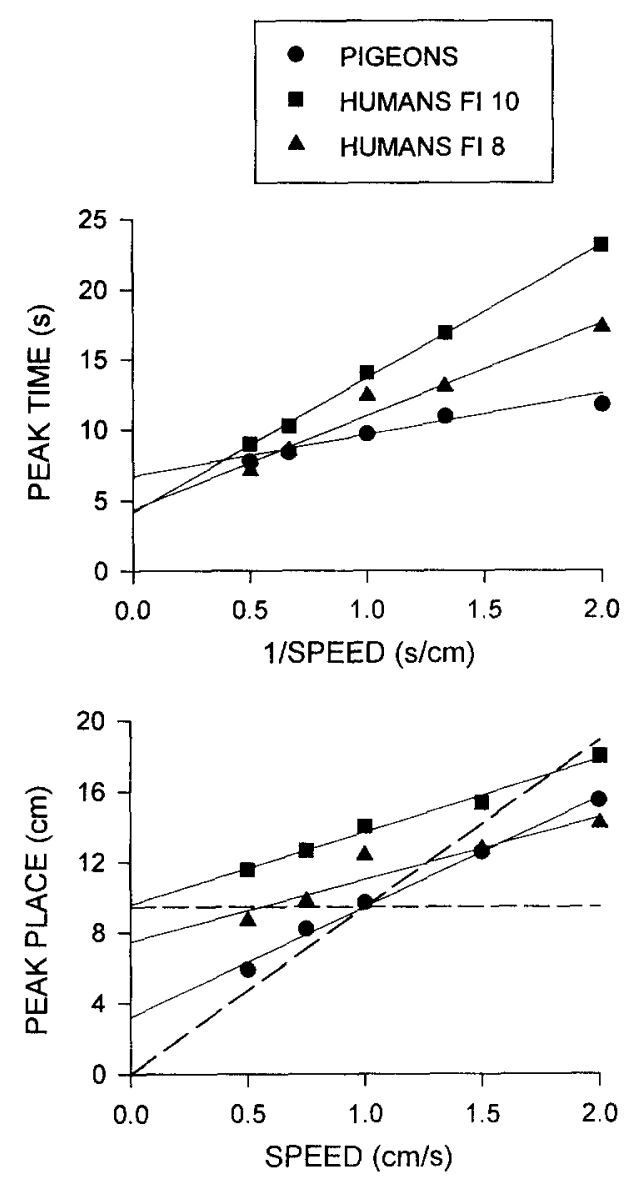

Figure 2. Peak times as a function of $1 /$ speed of moving landmark (top panel) and peak places as a function of speed of moving landmark (bottom panel) for pigeons and humans. Peak places were derived by calculating the position of the moving landmark at peak time. The solid lines show best-fitting (least squares criterion) regression lines through each set of five data points. The dotted lines show the predicted results for one set of data based on a strategy of using a spatial criterion alone (the flat line) or temporal criterion alone (the sloped line). FI = fixed interval.

tions. The results in all cases clearly reject both these hypotheses and suggest the use of both time and place in determining criterion.

\section{Weights for Space and Time}

We can calculate the weights that need to be assigned to time and place in order to produce the linear data in Figure 2. Consider the bottom panel of Figure 2 showing peak places and take the pigeon data as an example. If responding were based solely on place, the slope would be 0 , and it is reasonable to assume that the intercept would be at the peak place for the control speed of $1 \mathrm{~cm} / \mathrm{s}$. Assuming the linearity of the data to be correct, a good estimate for this value is the predicted peak time at speed $1 \mathrm{~cm} / \mathrm{s}$. According to the appropriate regression line in Figure 2, regression slope $x$ $1 \mathrm{~cm} / \mathrm{s}+$ regression intercept, or $6.23 \mathrm{~s} \times 1 \mathrm{~cm} / \mathrm{s}+3.20$ $\mathrm{cm}=9.43 \mathrm{~cm}$. We can then calculate peak places corresponding to constant peak times across speeds. These values lie on a line with an intercept of $0.00 \mathrm{~s}$ and a slope of $9.43 \mathrm{~s}$. That is, for constant peak times across speeds, peak place is a constant proportion of speed, with the proportionality constant estimated by the predicted peak place at speed $1 \mathrm{~cm} / \mathrm{s}(9.43 \mathrm{~cm} /[1 \mathrm{~cm} / \mathrm{s}]=9.43 \mathrm{~s})$. The weight accorded to time in the data then is the obtained slope (from the regression analysis) divided by the slope for the predicted line based on constant peak time, or $6.23 \mathrm{~s} / 9.43 \mathrm{~s}=0.66$. The weight accorded to place is, by necessity, 1 minus the weight accorded to time, or $1-0.66=0.34$. That is, the obtained slope is $66 \%$ of the way between the slope for a line predicted from place alone and the slope for a line predicted from time alone. A completely analogous analysis can be done on all the other lines shown in Figure 2.

Only the slopes and intercepts given in Table 1 are necessary for this task because this analysis merely transforms the two free parameters of a straight line into two other parameters. For peak places, the $y$ intercept (predicted value at $0 \mathrm{~cm} / \mathrm{s}$ ) is replaced by the predicted value at $1 \mathrm{~cm} / \mathrm{s}$, and the slope is replaced by a relative weight accorded to time ( 0.66 for pigeons) multiplied by the slope for the line predicted from time alone ( $9.43 \mathrm{~s}$ for pigeons). When using the least squares criterion, the predicted results of this exercise are exactly the predicted results of the regression analysis. The reader can verify that for peak times, the relative weights for place for pigeons, humans in the FI 10-s group, and humans in the FI 8-s group are, respectively, $0.30,0.69$, and 0.60 . For peak places, the relative weights for place for the three groups are, respectively, 0.34, 0.70 ,

Table 1

Summary of Analyses of Variance and Trend Analyses on Peak Times and Peak Places for Humans and Pigeons

\begin{tabular}{|c|c|c|c|}
\hline \multirow[b]{2}{*}{ Experiments } & \multirow[b]{2}{*}{ Pigeons } & \multicolumn{2}{|c|}{ Humans } \\
\hline & & FI 10 & FI 8 \\
\hline \multicolumn{4}{|c|}{ Peak times } \\
\hline Conditions & & & \\
\hline $\begin{array}{l}F \\
d f\end{array}$ & 19.09 & 58.95 & $\begin{array}{l}19.34 \\
4.25\end{array}$ \\
\hline \multicolumn{4}{|l|}{ Linear trend } \\
\hline$F$ & 71.60 & 235.48 & 74.38 \\
\hline$d f$ & 1,15 & 1,25 & 1,25 \\
\hline Regression slope $(\mathrm{cm})$ & 2.92 & 9.48 & 6.61 \\
\hline Regression intercept (s) & 6.71 & 4.18 & 4.36 \\
\hline$\%$ variance & 95.00 & 99.33 & 96.93 \\
\hline \multicolumn{4}{|c|}{ Peak places } \\
\hline Conditions & & & \\
\hline$F$ & 23.45 & 3.98 & 7.10 \\
\hline$d f$ & 4,15 & 4,25 & 4,25 \\
\hline \multicolumn{4}{|l|}{ Linear trend } \\
\hline$F$ & 93.14 & 14.95 & 25.18 \\
\hline$d f$ & I, 15 & 1,25 & 1,25 \\
\hline Regression slope (s) & 6.23 & 4.12 & 3.53 \\
\hline Regression intercept $(\mathrm{cm})$ & 3.20 & 9.57 & 7.46 \\
\hline$\%$ variance & 99.45 & 99.06 & 90.38 \\
\hline
\end{tabular}

Note. The $\%$ variance refers to the percent variance in the five averaged data points accounted for by the regression line. FI = fixed interval. 
and 0.68. Choosing for each group the graph that is most linear (i.e., of the peak-time vs. peak-place data, picking the set that accounts for the most variance), we obtain these weights for place: 0.34 for pigeons, 0.69 for humans in the FI 10-s group, and 0.60 for humans in the FI 8-s group.

\section{Subsidiary Data Analysis}

One possibility is that the individuals responded on the basis of spatial position only on some trials, whereas responding on the basis of time only on other trials. This behavior should produce double-peaked distributions. Response distributions on the extreme conditions shown in Figure 3, however, show single-peaked functions. For pigeons, data from individuals resemble the pooled pattern. For humans, data from individuals are too sparse to generate meaningful distributions. To pool across the data from in-
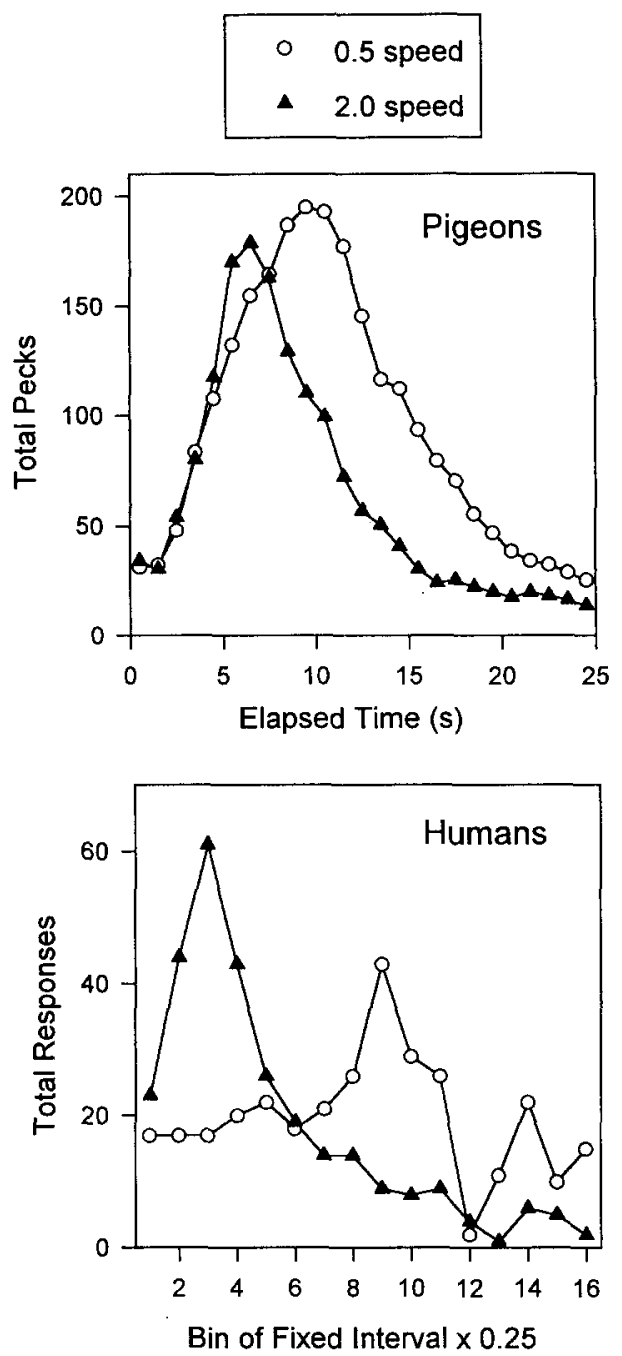

Figure 3. Pooled response distributions for pigeons (top panel) and humans (bottom panel) in the $0.5 \mathrm{~cm} / \mathrm{s}$ and $2 \mathrm{~cm} / \mathrm{s}$ speeds of landmark movement, summed across participants and sessions. dividuals who had different FIs, the data for humans in Figure 3 are presented in bins scaled to one quarter of the FI. Other ways of pooling the data produced similar results. For example, the data still looked single-peaked when each individual's distribution was scaled as a proportion of that individual's total responses before averaging, or when distributions were plotted in bins scaled to one fifth of each individual's median on control tests (data not shown).

We also conducted a trial-by-trial analysis on the pigeon data to further differentiate between different hypotheses about how the birds were using both time and space as bases for behavior. The tests from the pigeons appear to show the break-run-break data found in the standard peak procedure (Cheng \& Westwood, 1993; Church et al., 1994), in which a period of high rate of responding (run) is sandwiched between periods of low rates of responding (break). The trial-by-trial analysis provides a start and an end to the run for each trial.

The analysis followed Church et al.'s (1994) methods with only minor modifications. Pecks from the beginning of the trial to three times the duration of the FI were included. If a gap of time greater than $5 \mathrm{~s}$ separated two pecks, all pecks following the gap were deleted from analysis. This served to eliminate pecks near the end of the trial, when a high rate of responding can be seen again on many trials. From the remaining pecks, we picked out a start and an end of the run phase to maximize this entity,

$$
D 2 \times(R 2-R)+D 1 \times(R-R I) .
$$

$D 2$ is the duration of the run phase, $D l$ is the duration of the two break phases, $R$ is the average response rate on the trial, $R 2$ is the average response rate during the run phase, and $R l$ is the average response rate during the break phases. The weighting by $D I$ and $D 2$ serves to eliminate runs based on a short burst of responding at a high rate. Variations on this method have been tried on other data sets, and all produced similar results.

We eliminated trials with runs that started after the 10 -s FI or the $10-\mathrm{cm}$ positional criterion (whichever came later) or runs that ended before the $10-\mathrm{s}$ FI or the $10-\mathrm{cm}$ positional criterion (whichever came first). Thus, any runs that spanned either the spatial or the temporal criterion were included. On average, $71 \%$ of the tests were included. The start and the stop of the run was used to calculate two other statistics: the middle (average of start and stop) and the spread (stop-start). The means and standard deviations across trials of these statistics, as well as the correlations between them, are shown in Table 2. The pattern of results obtained in the trial-by-trial analysis here resembles what is found in the standard peak procedure (e.g., Cheng \& Westwood, 1993; Cheng et al., 1993; Church et al., 1994; Gibbon \& Church, 1990).

Yet another prediction comes from the hypothesis that some trials were based on spatial criterion only and some trials on temporal criterion only. The middles of runs ought to show a double-peaked distribution. The relevant data, shown in Figure 4 for the extreme speeds, do not show double-peaked distributions. Again, individual data resemble the grouped pattern. 
Table 2

Means, Standard Deviations, and Correlations Between Starts, Stops, Middles, and Spreads for Pigeons

\begin{tabular}{|c|c|c|c|c|c|c|c|c|c|c|}
\hline \multirow[b]{3}{*}{ Measure } & \multicolumn{10}{|c|}{ Speed $(\mathrm{cm} / \mathrm{s})$} \\
\hline & \multicolumn{2}{|c|}{0.50} & \multicolumn{2}{|c|}{0.75} & \multicolumn{2}{|c|}{1.00} & \multicolumn{2}{|c|}{1.50} & \multicolumn{2}{|c|}{2.00} \\
\hline & $M$ & $S D$ & $M$ & $\overline{S D}$ & $M$ & $S D$ & $M$ & $S D$ & $M$ & $S D$ \\
\hline Start & 4.73 & 2.59 & 4.69 & 2.68 & 4.20 & 2.49 & 4.64 & 3.17 & 4.70 & 3.49 \\
\hline Stop & 18.11 & 3.68 & 15.98 & 2.70 & 14.59 & 2.72 & 14.38 & 4.25 & 15.39 & 4.83 \\
\hline Middle & 11.42 & 2.40 & 10.34 & 2.09 & 9.41 & 2.04 & 9.51 & 3.15 & 9.80 & 3.63 \\
\hline Spread & 13.38 & 4.18 & 11.28 & 3.40 & 10.40 & 3.47 & 9.74 & 4.14 & 10.68 & 4.30 \\
\hline \multicolumn{11}{|c|}{ Correlations } \\
\hline Start-Stop & \multirow{2}{*}{\multicolumn{2}{|c|}{$\begin{array}{r}.15 \\
65\end{array}$}} & \multirow{2}{*}{\multicolumn{2}{|c|}{$\begin{array}{l}.22 \\
77\end{array}$}} & \multirow{2}{*}{\multicolumn{2}{|c|}{$\begin{array}{r}.19 \\
76\end{array}$}} & \multirow{2}{*}{\multicolumn{2}{|c|}{.42}} & \multicolumn{2}{|c|}{.49} \\
\hline Start-Middle & & & & & & & & & \multirow{2}{*}{\multicolumn{2}{|c|}{$\begin{array}{r}.81 \\
-.24\end{array}$}} \\
\hline Start-Spread & \multicolumn{2}{|c|}{-.48} & \multicolumn{2}{|c|}{$\begin{array}{r}.77 \\
-.62\end{array}$} & \multicolumn{2}{|c|}{-.63} & \multicolumn{2}{|c|}{-.30} & & \\
\hline Stop-Middle & \multicolumn{2}{|c|}{.84} & \multicolumn{2}{|c|}{.77} & \multicolumn{2}{|c|}{.75} & \multicolumn{2}{|c|}{.87} & \multicolumn{2}{|c|}{.24} \\
\hline Stop-Spread & \multicolumn{2}{|c|}{.078} & \multicolumn{2}{|c|}{.61} & \multicolumn{2}{|c|}{.59} & \multicolumn{2}{|c|}{.01} & \multicolumn{2}{|c|}{.70} \\
\hline Middle-Spread & \multicolumn{2}{|c|}{.33} & \multicolumn{2}{|c|}{.00} & \multicolumn{2}{|c|}{-.04} & \multicolumn{2}{|c|}{.29} & \multicolumn{2}{|c|}{.34} \\
\hline
\end{tabular}

Note. Means and standard deviations were measured in seconds. Standard deviations were calculated for tests obtained in each condition for each individual and then averaged across subjects.

The trial-by-trial analysis also allows us to rule out the possibility that the birds used two criteria for responding on a test, one based on spatial position and one based on time. If this were the case, the spreads ought to be larger than expected in the extreme speeds, in which the two criteria are farther apart. Because of scalar properties found in timing, we should expect spreads to be proportional to middles. Hence, the appropriate statistic to examine is spread/middle. Across the five conditions from the speed of 0.5 to $2.0 \mathrm{~cm} / \mathrm{s}$, this statistic was, respectively, $1.18,1.10,1.11,1.04$, and 1.10. Whereas these values differ significantly across conditions, $F(4,15)=3.84, p<.05$, they did not show the predicted quadratic trend.

Another concern was that the disappearance of the moving target off the right side of the screen might have affected behavior. At the fastest test speed, for example, the landmark disappeared off of the screen after approximately $13.5 \mathrm{~s}$. It is conceivable that individuals might either stop responding to the target when the landmark disappears or alternately use the disappearance of the landmark as a cue to respond. Inspection of the data shown in Figure 3, however, suggests that the disappearance of the landmark did not affect the shape of the response distributions in any important way. For example, the peak of responding for pigeons on the fastest speed (2.0) was well before $13.5 \mathrm{~s}$, and responding was already quite low before that time period elapsed. Similarly, for human participants the main peak of responding at the $2.0-\mathrm{s}$ speed occurred well before the stimulus disappeared from the screen (at Bin 6 for some participants and Bin 7 for the other participants). The shape of the distribution at the slowest test speed (0.5) could not be influenced by landmark disappearance because the landmark did not disappear before the end of those trials. We conclude that the landmark's disappearance did not have a major influence on responding and was not responsible for the difference in peaks between the fastest and slowest test speeds.
A number of human participants reported a strategy of attempting to use the spatial position of the moving landmark as a basis for responding, lending more evidence to the hypothesis that spatial position rather than landmark disappearance had the major influence on behavior. Nevertheless, the peak places of most participants were not constant in space. In fact, only 1 participant out of 12 showed constant peak places (values in five conditions within a $1-\mathrm{cm}$ range).

In summary, on the basis of averaged distributions and trial-by-trial analysis, the best conclusion we can make is that the birds were using a single criterion for responding on tests. This criterion was based on averaging a spatial posi-

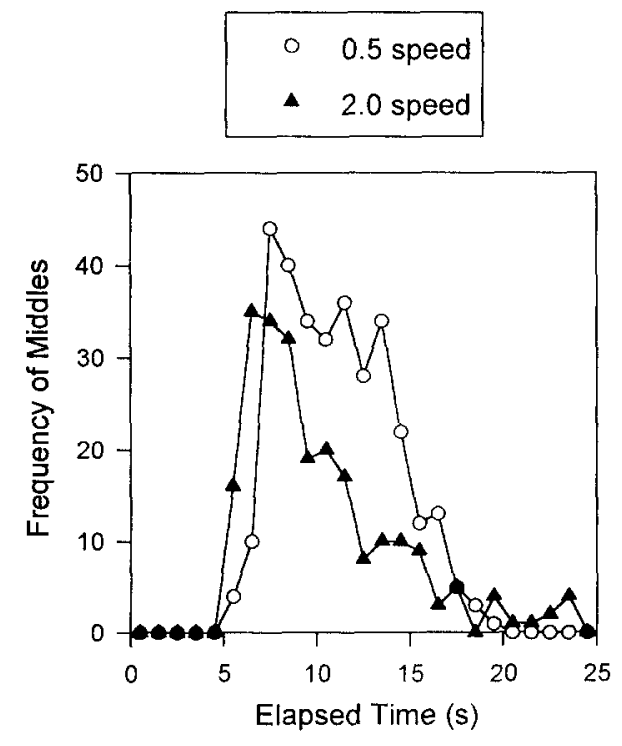

Figure 4. The distribution of middles for pigeons for the 0.5 $\mathrm{cm} / \mathrm{s}$ and $2 \mathrm{~cm} / \mathrm{s}$ speeds of landmark movement, totaled across participants. 
tion with a duration of time. With the humans, the data were too sparse for any trial-by-trial analysis. However, on the basis of grouped data, the best conclusion is also that, similar to the pigeons, the humans averaged a spatial criterion and a temporal criterion to determine responding on each test.

\section{Discussion}

In this experiment, humans and pigeons were presented with situations in which behavior can be based on two redundant sources of information, either the time elapsed since a landmark started moving or the spatial position reached by the landmark. Both species used both types of information in responding, although the relative weighting of time and place appeared to differ, with pigeons giving relatively more weight to the temporal dimension. Most important, however, subsidiary analyses of the data suggested that for both species, two sources of radically different sensory information are combined in an interesting way. If our interpretation is correct, the two sources of information had been combined to derive a single target time-place or duration-position, which served as the criterion around which responses were made. The averaging of time and space did not appear to be a case of two separate systems vying for control over the same motor systems. Rather, the two sources of information, one based on visual motion perception and one based on an internal clock, appeared to have been combined centrally to form a single set of commands for the motor systems.

Our analysis of data shows that humans put more weight on place than on time, both absolutely and relative to the pigeons. We do not draw too much from this point, as we have data here from only one test situation. Perhaps the assignment of weights to time and place depends much on testing conditions. One reviewer indicated that when the pigeons were pecking at the target, it probably was difficult to clearly perceive the moving landmark above. This may give an explanation as to why the birds weighted time relatively more. The important conclusion to draw is that both species averaged time and place in creating a single criterion for responding.

\section{Neurophysiological Considerations}

It is interesting that a value in the brain representing elapsed time can be averaged in some fashion with a value representing spatial position. But this has further implications and suggests at least the following two points. (a) The brain actually stores values that represent different dimensions of experience, such as spatial location and elapsed time. (b) Such values must be translatable to some common code that allows averaging to take place.

With regard to point (a), the neurophysiological basis (or bases) for storing values of dimensions of experience, if this phrase is indeed the right way of framing this reduction problem, remains a mystery and a central challenge for neuroscience. If storing of values of dimensions is what learning and memory is all about, it suggests constraints on mechanisms to look for in solving this reduction problem (Gallistel, 1990). With regard to point (b), one tantalizing possibility is that all information stored in the brain to represent the world might be coded in a common way. In this scheme, time and space can be averaged because the basis for coding numerical values in time and space is the same. Separate codes would indicate the units (e.g., s, cm, etc.) attached to values. A common basis of coding information would certainly be good news to those searching for the neurophysiological basis of memory because it limits the mechanism sought to one rather than many mechanisms.

This common coding hypothesis is not empty speculation. It can generate testable behavioral predictions. If time and space are coded in a common fashion, it ought to be easier for a creature to learn a congruent time-space mapping than an incongruent mapping. To use a concrete example, it would be easier to map a short duration and a short distance onto one response and a long duration and a long distance onto another response. It would be more difficult to map a short duration and a long distance onto one response, and a long duration and a short distance onto another response. Of course, many such predictions would need to be tested because many conjectures can produce the obtained results in any particular set of experiments.

\section{Averaging Outputs of Different Systems}

The averaging of different sources of information to determine a single criterion for behavior is not unique to our task. We have already reviewed in the introduction how different cues may be averaged within a system such as interval timing or landmark-based spatial memory. One good example of the averaging of information on the basis of different systems is the work of Etienne and colleagues on the use of landmark and inertial information in hamsters (Etienne, Joris-Lambert, Dahn-Hurni, \& Reverdin, 1995; Etienne, Joris-Lambert, Maurer, Reverdin, \& Sitbon, 1995; Etienne, Teroni, Hurni, \& Portenier, 1990). In these tasks, the hamsters lived in a home just outside a circular arena. They were induced to go to the center of the arena to collect food for hoarding. On some test trials, the landmarks around the arena were rotated by $90^{\circ}$. This puts two systems of spatial orientation at variance. One system is piloting or landmark-based spatial memory, which is based on coding the goal location in terms of spatial relations to surrounding landmarks. The other system is inertial navigation or path integration, which is based on keeping track of the path covered by the subject from the start of the journey (see Gallistel, 1990, chap. 4). Many hamsters under such circumstances struck out in a direction homeward somewhere intermediate between the dictates of the landmarks and the dictates of path integration.

In Etienne et al.'s (1990, 1995a, 1995b) hamsters, the dictates of both systems, landmark-based spatial memory and path integration, are couched in spatial terms. In our task, the dictates of the two sources of information are couched in terms of spatial position and elapsed time, two 
radically different dimensions of experience. We suspect that the central integration of different types of information is a notable feature of neurocognitive architecture.

\section{References}

Cheng, K. (1988). Some psychophysics of the pigeon's use of landmarks. Journal of Comparative Physiology A, 162, 815826.

Cheng, K. (1989). The vector sum model of pigeon landmark use. Journal of Experimental Psychology: Animal Behavior Processes, 15, 366-375.

Cheng, K. (1992). Three psychophysical principles in the processing of spatial and temporal information. In W. K. Honig \& J. G. Fetterman (Eds.), Cognitive aspects of stimulus control (pp. 69-88). Hillsdale, NJ: Erlbaum.

Cheng, K. (1994). The determination of direction in landmarkbased spatial search in pigeons: A further test of the vector sum model. Animal Learning and Behavior, 22, 291-301.

Cheng, K., \& Roberts, W. A. (1989). Timing multimodal events in pigeons. Journal of the Experimental Analysis of Behavior, 52, 363-376.

Cheng, K., \& Roberts, W. A. (1991). Three psychophysical principles of timing in pigeons. Learning and Motivation, 22, 112 128.

Cheng, K., \& Sherry, D. (1992). Landmark-based spatial memory in birds (Parus atricapillus and Columba livia): The use of edges and distances to represent spatial positions. Journal of Comparative Psychology, 106, 331-341.

Cheng, K., \& Westwood, R. (1993). Analysis of single trials in pigeons' timing performance. Journal of Experimental Psychology: Animal Behavior Processes, 19, 56-67.

Cheng, K., Westwood, R., \& Crystal, J. D. (1993). Memory variance in the peak procedure of timing in pigeons. Journal of Experimental Psychology: Animal Behavior Processes, 19, 6876.

Church, R. M., Meck, W. H., \& Gibbon, J. (1994). Application of scalar timing theory to individual trials. Journal of Experimental Psychology: Animal Behavior Processes, 20, 135-155.

Etienne, A. S., Joris-Lambert, S., Dahn-Hurni, C., \& Reverdin, B. (1995a). Optimizing visual landmarks: 'Two and three dimensional minimal landscapes. Animal Behaviour, 49, 165-179.

Etienne, A. S., Joris-Lambert, S., Maurer, R., Reverdin, B., \& Sitbon, S. (1995b). Optimizing distal landmarks: Horizontal versus vertical structures and relation to background. Behavioural Brain Research, 68, 103-116.

Etienne, A. S., Teroni, E., Hurni, C., \& Portenier, V. (1990). The effect of a single light cue on homing behaviour of the golden hamster. Animal Behaviour, 39, 17-41.

Fetterman, J. G. (1993). Numerosity discrimination: Both time and number matter. Journal of Experimental Psychology: Animal Behavior Processes, 19, 149-164.

Gallistel, C. R. (1990). The organization of learning. Cambridge, MA: Massachusetts Institute of Technology Press.

Gibbon, J., \& Church, R. M. (1990). Representation of time. Cognition, 37, 23-54.

Grandage, A. (1958). Orthogonal coefficients for unequal intervals. Biometrics, 14, 287-289.

Hinton, S. C., Gibbon, J., Rakitin, B. C., \& Meck, W. H. (1993, November). Effects of feedback on human interval timing. Paper presented at the meeting of the Psychonomic Society, Washington, DC.

Meck, W. H., \& Church, R. M. (1983). A mode control model of counting and timing processes. Journal of Experimental Psychology: Animal Behavior Processes, 9, 320-334.

Roberts, S. (1981). Isolation of an internal clock. Journal of Experimental Psychology: Animal Behavior Processes, 7, 242268.

Roberts, W. A., Cheng, K., \& Cohen, J. S. (1989). Timing visual and auditory signals in pigeons. Journal of Experimental Psychology: Animal Behavior Processes, 15, 23-35.

Spetch, M. L., Cheng, K., \& Mondloch, M. V. (1992). Landmark use by pigeons in a touch-screen spatial search task. Animal Learning and Behavior, 20, 281-292.

Wearden, J. H. (1991). Do humans possess an internal clock with scalar timing properties? Learning and Motivation, 22, 59-83.

Wearden, J. H. (1995). Categorical scaling of stimulus duration by humans. Journal of Experimental Psychology: Animal Behavior Processes, 21, 318-330.

Wearden, J. H., \& McShane, B. (1988). Interval production as an analogue of the peak procedure: Evidence for similarity of human and animal timing processes. Quarterly Journal of Experimental Psychology, 40B, 363-375.

Received May 22, 1995

Revision received August 1, 1995 Accepted November 7, 1995 